Systems/Circuits

\title{
Clinically Anxious Individuals Show Disrupted Feedback between Inferior Frontal Gyrus and Prefrontal-Limbic Control Circuit
}

\author{
Jiook Cha, ${ }^{1 *}$ Daniel DeDora, ${ }^{2,3 *}$ Sanja Nedic, ${ }^{2,3}$ Jaime Ide, ${ }^{2,3}$ Tsafrir Greenberg, ${ }^{4}$ Greg Hajcak, ${ }^{4}$ \\ and Dilianne Rivka Mujica-Parodi ${ }^{2,3}$ \\ ${ }^{1}$ Department of Psychiatry, Columbia University Medical Center and the New York State Psychiatric Institute, New York, New York 10032, ${ }^{2}$ Department of \\ Biomedical Engineering, Stony Brook University School of Medicine, Stony Brook, New York 11794, ${ }^{3}$ Department of Radiology, A. A. Martinos Center for \\ Biomedical Imaging, Massachusetts General Hospital, Charlestown, Massachusetts 02129, and ${ }^{4}$ Department of Psychology, Stony Brook University, Stony \\ Brook, New York 11794
}

Clinical anxiety is associated with generalization of conditioned fear, in which innocuous stimuli elicit alarm. Using Pavlovian fear conditioning (electric shock), we quantify generalization as the degree to which subjects' neurobiological responses track perceptual similarity gradients to a conditioned stimulus. Previous studies show that the ventromedial prefrontal cortex (vmPFC) inversely and ventral tegmental area directly track the gradient of perceptual similarity to the conditioned stimulus in healthy individuals, whereas clinically anxious individuals fail to discriminate. Here, we extend this work by identifying specific functional roles within the prefrontal-limbic circuit. We analyzed fMRI time-series acquired from 57 human subjects during a fear generalization task using entropic measures of circuit-wide regulation and feedback (power spectrum scale invariance/autocorrelation), in combination with structural (diffusion MRI-probabilistic tractography) and functional (stochastic dynamic causal modeling) measures of prefrontal-limbic connectivity within the circuit. Group comparison and correlations with anxiety severity across 57 subjects revealed dysregulatory dynamic signatures within the inferior frontal gyrus (IFG), which our prior work has linked to impaired feedback within the circuit. Bayesian model selection then identified a fully connected prefrontal-limbic model comprising the IFG, vmPFC, and amygdala. Dysregulatory IFG dynamics were associated with weaker reciprocal excitatory connectivity between the IFG and the vmPFC. The vmPFC exhibited inhibitory influence on the amygdala. Our current results, combined with our previous work across a threatperception spectrum of 137 subjects and a meta-analysis of $366 \mathrm{fMRI}$ studies, dissociate distinct roles for three prefrontal-limbic regions, wherein the IFG provides evaluation of stimulus meaning, which then informs the vmPFC in inhibiting the amygdala.

Key words: connectivity; control systems; dynamic causal modeling; functional MRI; inferior frontal gyrus; prefrontal cortex

Significance Statement

Affective neuroscience has generally treated prefrontal regions (orbitofrontal cortex, dorsolateral prefrontal cortex, inferior frontal gyrus, ventromedial prefrontal cortex) equivalently as inhibitory components of the prefrontal-limbic system. Yet research across the anxiety spectrum suggests that the inferior frontal gyrus may have a more complex role in emotion regulation, as this region shows abnormal function in disorders of both hyperarousal and hypoarousal. Using entropic measures of circuitwide regulation and feedback, in combination with measures of structural and functional connectivity, we dissociate distinct roles for three prefrontal-limbic regions, wherein the inferior frontal gyrus provides evaluation of stimulus meaning, which then informs the ventromedial prefrontal cortex in inhibiting the amygdala. This reconfiguration coheres with studies of conceptual disambiguation also implicating the inferior frontal gyrus.

\section{Introduction}

Anxiety disorders have been linked with flawed evaluation of threat (Greenberg et al., 2013a; Cha et al., 2014b), in which in- nocuous stimuli elicit neurobiological and behavioral fear responses. This hypersensitivity to threat may be driven by diminished recruitment of the lateral prefrontal cortex during cog- 
nitive and emotional tasks (Bishop et al., 2004; Bishop, 2009; Strawn et al., 2012). The inferior frontal gyrus (IFG) is a subregion of the lateral prefrontal cortex that plays an important role in the regulation of emotion and attention (Aron et al., 2003; Ochsner and Gross, 2005; Depue et al., 2007; Sagaspe et al., 2011; Ochsner et al., 2012; Vanderhasselt et al., 2013), including modulation of the threat response (Fitzgerald et al., 2006; Eippert et al., 2007).

Previously, we showed that IFG (Brodmann's area 45) reactivity to fearful faces positively correlated with suppression of the amygdala responses, and negatively correlated with trait anxiety in healthy individuals (Mujica-Parodi et al., 2009). Later, we quantified the functional dynamics of the IFG in trait anxious individuals with power spectrum scale invariance (PSSI) (Tolkunov et al., 2010), a control systems-derived measure of circuit-wide regulation. Optimal regulation, as required for maintenance of biological allostasis, requires that a circuit not only be supple enough to effectively respond to stimuli, but also be constrained enough by negative feedback to efficiently return the system to baseline. This critical balance, between excitatory and inhibitory components of a negative feedback loop, produces outputs whose dynamics can be characterized as "pink noise" (Rădulescu and Mujica-Parodi, 2014). Our PSSI analyses showed that individuals with greater trait anxiety exhibit IFG functional dynamics that shift away from pink noise to a more chaotic "white noise." However, the underlying basis for the dysregulation is not well understood, as white noise can result either from stronger chaotic excitatory inputs or weaker negative feedback or both (Rădulescu and Mujica-Parodi, 2014). Recently, we observed the same pattern of aberrant IFG functional dynamics in a high sensationseeking group of first-time skydivers with poor threat evaluation (Mujica-Parodi et al., 2014). The commonality in the IFG results across the two extreme ends of the anxiety spectrum in healthy individuals, both of which share deficits in threat evaluation, raises the question of whether our (Mujica-Parodi et al., 2009; Rădulescu and Mujica-Parodi, 2014) and others' (Aron et al., 2003; Bishop et al., 2004; Ochsner and Gross, 2005; Fitzgerald et al., 2006; Depue et al., 2007; Eippert et al., 2007; Bishop, 2009; Sagaspe et al., 2011) initial view of the IFG as simply an inhibitory component of the negative feedback loop regulating emotion might be incomplete.

Here, we focus on clarifying three critical aspects of the IFG's role within the prefrontal-limbic control circuit responsible for threat evaluation, with the aim of better informing our previous results, as well as better interpreting the region's role across the emotion literature. First, do our trait anxiety results (aberrant IFG dynamics) continue to hold when we move to clinical anxiety? Second, if so, does the IFG provide the direct inhibitory component of the prefrontal-limbic control system, as we and others initially suggested, or does it function in a distinct (perhaps evaluative) capacity within the circuit, as suggested by our later findings and the wider, nonaffective, fMRI literature on that area? Third, do the aberrant dynamics first observed in trait anxiety reflect hyperexcitatory function and/or weaker regulation across the circuit?

To address these three questions, we further analyzed data acquired from a fear generalization task that we have previously shown to be capable of probing subtle features of threat disambiguation in clinical anxiety (Greenberg et al., 2013a; Cha et al., 2014b). For this study, we recruited medication-free individuals with clinical anxiety (generalized anxiety disorder [GAD]) and age- and gender-matched controls to take part in a fear-generalization paradigm (Greenberg et al., 2013b), which
Table 1. Demographic and clinical data for individuals with GAD and healthy controls $^{a}$

\begin{tabular}{lcclc}
\hline Group & GAD & Healthy control & $t$ & $p$ \\
\hline Age (SD) & $22.3(5.2)$ & $21.5(5.2)$ & 0.63 & 0.58 \\
Weight & $130.0(18.8)$ & $142.2(27.0)$ & 1.93 & 0.06 \\
MASQ-GDA & $26.5(7.4)$ & $16.8(4.1)$ & 5.73 & $<0.0001$ \\
MASQ-DD & $35.5(12.1)$ & $17.9(3.6)$ & 6.81 & $<0.0001$ \\
MASQ-AA & $28.8(9.2)$ & $20.8(3.9)$ & 4.03 & 0.0002 \\
MASQ-AD & $76.0(15.4)$ & $53.3(11.6)$ & 6.01 & $<0.0001$ \\
\hline
\end{tabular}

${ }^{a} \mathrm{DD}$, Distress depression; $\mathrm{AA}$, anxious arousal; $\mathrm{AD}$, anhedonic depression.

included stimuli that were threat-ambiguous due to their perceptual similarity to a conditioned stimulus (CS). Our prior work explored traditional activation-based measures in the context of fear generalization (Greenberg et al., 2013a; Cha et al., 2014b). Here, we build upon this work: first, we use PSSI to test for dysregulatory functional dynamics along the entire time-series; then, we use functional and structural connectivity analyses, including stochastic dynamic causal modeling (sDCM), to further interpret our results.

\section{Materials and Methods}

\section{Study design}

This dataset includes 57 participants (age, mean \pm SD, $22.3 \pm 4.5$ years; Table 1), who took part in a fear generalization task while being scanned. Of these, 32 were individuals with GAD and 25 were healthy controls. There is a significantly higher occurrence of anxiety disorders in females than males (McLean et al., 2011); thus, we tested only females for the study to avoid potentially uneven sample sizes due to lack of male participants. This study was approved by the Stony Brook University Institutional Review Board; all participants provided informed consent.

\section{Diagnoses}

Clinical diagnoses were performed in two steps: informal clinical interview and a structured clinical interview. Diagnoses were based on DSM-IV Axis I Disorders (American Psychiatric Association, 2000; First et al., 2002). The control group was cleared of Axis I diagnoses, whereas the patient group was diagnosed with GAD; 17 of the 32 GAD patients were diagnosed with comorbid GAD and major depressive disorder. All participants were free from psychiatric medication for at least 6 months leading up to the experiment; additional exclusion criteria included presence of another major psychiatric illness, such as bipolar disorder, alcohol or substance dependence, and schizophrenia. All participants completed several self-report questionnaires, including the Mood and Anxiety Symptom Questionnaires (MASQ) (Watson et al., 1995), presented in Table 1.

\section{fMRI task}

After screening and consenting in accordance with a protocol approved by the Stony Brook University Institutional Review Board, participants read written instructions for the entire study. The fear generalization task was administered inside the fMRI scanner. Before the task, a voltage level was set for each participant to a level that was "uncomfortable but not painful" and was delivered to the left forearm (Constant Voltage Stimulator STM 200; Biopac Systems). Instructions for the task were then provided again verbally. Participants were told that a mid-sized rectangle (CS) indicated a $50 \%$ probability that they would receive a subsequent electric shock but that shocks would never follow rectangles of greater or lesser size. Following the instruction, we administered a conditioning phase. This included five presentations of the CS with the shock (i.e., $100 \%$ probability) and a single presentation of each generalized stimulus (GS) in a pseudo-random order with interstimulus intervals of $4-10 \mathrm{~s}$. A generalization phase immediately followed. The fear generalization task (Fig. 1) consisted of the presentation of an initial fixation screen (a white cross on a black background), followed by a $2 \mathrm{~s}$ stimulus presentation. For the CS, a red rectangle was paired with $500 \mathrm{~ms}$ of electric shock with a partial reinforcement schedule of a 50\% delivery probability, $1500 \mathrm{~ms}$ 
after the cue onset. For GS, we used six red rectangles with systematically varying widths (i.e., $\pm 20 \%$, $\pm 40 \%$, and $\pm 60 \%$ ) without shock. Each GS was pseudo-randomly presented 15 times, and the CS was presented 30 times (15 with shock and 15 without shock), for a total of 120 trials. Trials were flanked with interstimulus intervals ranging from 4 to $10 \mathrm{~s}$ with a white fixation crosshair on a black background. Task duration was $15 \min 24 \mathrm{~s}$.

\section{MRI data acquisition}

We scanned participants with a 3T Siemens Magnetom Trio scanner within the Social, Cognitive, and Affective Neuroscience Center at Stony Brook University. We acquired a total of $440 T_{2}^{*}$-weighted echo planar images with an oblique coronal angle and $\mathrm{TR}=2100 \mathrm{~ms}, \mathrm{TE}=$ $23 \mathrm{~ms}$, flip angle $=83^{\circ}$, matrix $=96 \times 96$, $\mathrm{FOV}=224 \times 224 \mathrm{~mm}$, slices $=37$, and slice thickness $=3.5 \mathrm{~mm}$. For structural scans, $T_{1^{-}}$ weighted images were acquired with the following parameters: $\mathrm{TR}=1900 \mathrm{~ms}, \mathrm{TE}=2.53$, flip angle $=9^{\circ}, \mathrm{FOV}=176 \times 250 \times 250 \mathrm{~mm}$, matrix $=176 \times 256 \times 256$, and voxel size $=$ $1 \times 0.98 \times 0.98 \mathrm{~mm}$. Diffusion tensor imaging (DTI) was collected in a separate session. We used the following parameters to collect DTI: TR = $5500 \mathrm{~ms}, \mathrm{TE}=93 \mathrm{~ms}, \mathrm{FOV}=220 \times 220 \mathrm{~mm}$, matrix $=120 \times 220 \times 220$, voxel size $=1.7 \times 1.7 \times 3.0 \mathrm{~mm}$, EPI factor $=128$, slices $=40$, slice thickness $=3 \mathrm{~mm}$, bandwidth $=1396 \mathrm{~Hz} /$ pixel, and GRAPPA acceleration factor $=2$. The series included two initial images acquired without diffusion weighting and with diffusion weighting along 40 noncollinear directions $\left(b=800 \mathrm{sm}^{-2}\right)$.

\section{MRI analyses}

Overview. In previous articles analyzing the same dataset, we reported standard activation results (task-based neural correlates of fear generalization) in GAD (Greenberg et al., 2013a; Cha et al., 2014b). Here, we focused on identifying key features of circuit regulation using systems-based analyses across the entire time-series. First, we examined circuit efficiency of the negative feedback loop using a measure of allostatic regulation, PSSI $\beta$-signatures (Rubin et al., 2013), from the fear generalization fMRI data. Complementing PSSI, we estimated autocorrelation of the same fMRI data to identify the specific timescales over which feedback occurs. Next, we tested our hypothesis that disrupted prefrontal-limbic regulation of negative affect (quantified by PSSI $\beta$-signatures) in the GAD group was linked to altered prefrontallimbic connectivity (Rădulescu and Mujica-Parodi, 2014), using three well-established connectivity analyses. We performed (1) psychophysiological interaction (PPI) analyses to identify prefrontal-limbic regions whose "correlational" connectivity with the IFG is associated with IFG PSSI $\beta$-signatures in a standard task-specific manner; (2) sDCM to define the prefrontal-limbic threat circuit whose directional connectivity with the IFG is associated with IFG PSSI $\beta$-signatures in a model-free approach; and (3) probabilistic diffusion tractography to investigate whether the integrity of a putative anatomical substrate of the IFG's functional connectivity with the prefrontal-limbic threat circuit is associated with IFG $\beta$-signatures. Therefore, by using both task-specific (PPI) and task-free analyses (DCM), we provide complementary functional connectivity estimates. The further integration of functional connectivity (PPI and DCM) and structural connectivity (tractography) analyses is designed to provide a mechanistic basis for interpreting PSSI $\beta$-signatures in the IFG.

\section{Preprocessing}

We performed standard preprocessing procedures for fMRI, including slice time correction, motion correction, normalization, and smoothing with a $6 \mathrm{~mm}$ FWHM Gaussian kernel in SPM8 (www.fil.ion. ucl.ac.uk/spm). In addition, our preprocessing procedures included de-
Table 2. Power law $\boldsymbol{\beta}$-signatures within the IFG are positively correlated with functional coupling (PPI) between the IFG and prefrontal-limbic regions (ROI analysis), as well as bilateral temporal lobes (whole-brain analysis)

\begin{tabular}{|c|c|c|c|c|c|c|}
\hline \multirow[b]{2}{*}{ Region } & \multirow{2}{*}{$\begin{array}{l}\text { Cluster extent } \\
\text { (no. of voxels) }\end{array}$} & \multirow[b]{2}{*}{ Peak $p$} & \multirow[b]{2}{*}{ Corrected $p$} & \multicolumn{3}{|l|}{ MNI } \\
\hline & & & & $x$ & $y$ & $z$ \\
\hline \multicolumn{7}{|l|}{ ROI analysis } \\
\hline Left ventromedial PFC & 125 & $4 \times 10^{-5}$ & $<0.005$ & -10 & 40 & -16 \\
\hline Right ventrolateral PFC & 97 & $7 \times 10^{-5}$ & $<0.01$ & 28 & 34 & -18 \\
\hline \multicolumn{7}{|l|}{ Whole-brain analysis } \\
\hline Right caudate & 143 & $8 \times 10^{-6}$ & $<0.001$ & -4 & 2 & 4 \\
\hline Left temporal lobe & 659 & $1 \times 10^{-6}$ & $<0.001$ & -66 & -46 & 4 \\
\hline Right temporal lobe & 210 & $3 \times 10^{-4}$ & $<0.001$ & 66 & -40 & -4 \\
\hline
\end{tabular}

trending and regression of global signal, CSF, white matter, and 6 degrees of motion (Rubin et al., 2013).

\section{Power spectrum scale invariance}

Using methods previously optimized by our group for fMRI (Rubin et al., 2013), we estimated the slope of the linear fit $(\beta)$ from each FFTtransformed time-series $S(f)$ as per $S(f) \propto f^{-\beta}$. Power spectrum densities were computed from preprocessed EPI images on a voxelwise basis and plotted on a log-log scale. We computed $\beta$ within a frequency window of $0.01-0.1 \mathrm{~Hz}$ using least-squares fitting; this range of frequencies has previously been shown to obey a power law model (Table 2) (He et al., 2010; He, 2011). Consistent with other groups, as well as our more recent publications using PSSI, here we used preprocessed time-series without taking the derivative and reported $\beta$ to simplify interpretation of correlations by having PSSI represented by positive numbers. Thus, $\beta=$ 0 represents a power spectrum with maximum entropy (white noise). As $\beta$ increases, a given data value is not only affected by its inputs, but also more strongly by previous outputs (i.e., previous data values). This nonlinear self-dependence is sometimes described as "fractality," "selfsimilarity," "memory," or "persistence," and is a key feature of all feedback loops. We had previously shown that persistence results from either diminished excitatory inputs or tighter homeostatic constraint over the system via negative feedback (Rădulescu and Mujica-Parodi, 2014). Group differences in $\beta$-signatures were examined using voxelwise $t$ tests. Independent variables included group and subject, whereas individual $\beta$ images were used as dependent variables. To rule out head motion-related artifacts on $\beta$-signatures (Rubin et al., 2013), we confirmed no group differences in movement (e.g., absolute maximum translation, maximum rotation, mean translation, mean rotation) (twosample $t$ test, $p$ values $>0.3$ ). We then tested for correlations between 
PSSI values and MASQ anxiety subscale (general distress anxiety [GDA]).

A voxelwise group comparison was performed on PSSI values using the IFG as an a priori ROI (Mujica-Parodi et al., 2009; Tolkunov et al., 2010). For multiple correction, we used a cluster extent-based method by estimating a minimum cluster size under a voxelwise $p$ threshold of 0.005 that corresponds to $\alpha$ of 0.05 within each hemisphere of the IFG. The IFG ROI was derived from an anatomical atlas (Tzourio-Mazoyer et al., 2002). Additionally, as exploratory analyses, we considered the vPFC (including the medial and lateral regions) and the amygdala, based on their well-established roles in fear and anxiety.

\section{Autocorrelation function}

To aid in the circuit-wide interpretation of PSSI, we also computed the temporal autocorrelation function (ACF) of preprocessed BOLD timeseries. Negative feedback loops produce outputs in the form of damped oscillations, as feedforward (excitatory) and feedback (inhibitory) components work in series (i.e., with some lag) to establish allostasis. As described above, closed-loop systems with greater negative feedback have more "memory" within their time-series, as outputs not only reflect inputs, but also previous outputs. The ACF reflects the correlation of a signal with itself at different time lags and thus provides more detailed information about the speed at which feedback occurs. Here, we modeled the ACF as a decaying exponential function with mean lifetime parameter $\tau(\mathrm{s})$, the system's "memory" (the amount of time a signal maintains an association with past and future values). A time-series from a system with stronger feedback exhibits greater persistence/memory and therefore longer mean lifetimes $\tau(\mathrm{s})$, whereas a time-series from a system under perturbation and no feedback exhibits greater chaos (white noise) with a $\tau$ (s) close to 0 .

PSSI and ACF are related via the Wiener-Khinchin theorem, which states that the Fourier transform of the ACF is equivalent to the power spectral density, with which PSSI is computed. The ACF augments PSSI analysis in two ways: (1) the autocorrelation is a function of time, and its mean lifetime can be expressed in units of seconds, making it more easily interpretable than PSSI $\beta$-signatures (which are expressed as $\partial(\log -$ (power $)) / \partial(\log ($ frequency $)))$; and (2) model fit is improved compared with PSSI, partially because of the use of least-squares fitting in log-log space in PSSI (Clauset et al., 2009).

We estimated voxelwise ACF using the Econometrics Toolbox implemented in MATLAB R2010a (MathWorks, Natick, MA). We then fit an exponential function of the form $y=\mathrm{ae}^{-\mathrm{bx}}$ to the first $9(\mathrm{lag}=8)$ points of each voxel's ACF using the nonlinear least-squares fitting method as implemented in the Curve Fitting Toolbox within MATLAB R2010a. We chose to model eight lags $(16.8 \mathrm{~s}$ at TR $=2.1 \mathrm{~s})$ because the canonical hemodynamic response function spans $16 \mathrm{~s}$ after each stimulus (Miezin et al., 2000); this duration was found to allow for full relaxation of ACF signal (Fig. $2 C$ ). Here, b values are related to the mean lifetime decay of a signal, $\tau(\mathrm{s})$, through the relation $\tau=1 / \mathrm{b} \times \mathrm{TR}$. Group differences in gray matter voxelwise $b$ values were examined using the same GLM approach and $t$ contrasts in SPM as in the PSSI group differences analysis. Finally, $\mathrm{ACF} b$ values were converted to mean half-life $\tau$ for interpretation. The size of $\tau$ determines the rate of decay of ACF, with lower $\tau$ signifying faster decay (more chaotic, therefore lower $\beta$ ) and higher $\tau$ signifying slower decay (more persistence/higher $\beta$ ). The entire procedure was limited to gray matter voxels to reduce computational time associated with nonlinear fitting. It is important to differentiate the temporal autocorrelation of a time-series reported here and the autocorrelation of the residuals following GLM or similar analyses; the latter refers to temporal prewhitening of a signal, a technique frequently used to satisfy the assumption of independence of errors in linear models (Woolrich et al., 2001).

\section{PPI}

We first examined IFG-seeded effective connectivity during fear generalization and its association with $\beta$-signatures using PPI analysis (Friston et al., 1997) in a standard task-specific manner. A seed region was defined based on the group differences in $\beta$-signatures within the left IFG. A representative time-series was extracted from the IFG by applying a 6 -mm-radius sphere centered at the peak $\beta$-signature group difference and deconvolved with the hemodynamic response function. This timeseries was multiplied by a regressor for all GS minus baseline, and then reconvolved with hemodynamic response function. This procedure generated one interaction term representing IFG functional coupling during all GS, as well as individual interaction terms for each condition. The interaction terms were entered into a separate model containing three regressors: the IFG time-series by "all GS versus baseline" interaction, "all GS versus baseline," and the unmodulated IFG time-series. We entered the demeaned IFG $\beta$-signatures and group as regressors. We then tested for correlations between IFG $\beta$ and functional coupling for the "all GS" condition within the ventral PFC (including medial and lateral) and amygdala. Results were corrected for multiple comparisons using a cluster extent approach based on AFNI's 3dClustSim (http://afni.nimh.nih. gov). For each ROI (an anatomical atlas of the vPFC, the orbitofrontal gyrus including the medial and lateral regions, and the amygdala); (Tzourio-Mazoyer et al., 2002), we estimated a minimum cluster size under a voxelwise $p$ threshold of 0.005 that corresponds to $\alpha$ of 0.05 . We also performed an exploratory voxelwise analysis to assess the relationship between IFG-seeded effective connectivity and IFG $\beta$-signatures across the whole brain.

\section{DCM}

Overview. We used DCM (Friston et al., 2003) to test various directional models of the circuit and to evaluate the relationship between the causal connectivity and IFG $\beta$-signatures. As a method, DCM identifies directed connectivity among the nodes; hidden underlying neural populations are modeled from measured hemodynamic activity and a system of differential equations is constructed to best explain the given behavior. Bayesian model selection (BMS) is used to select the most likely model from a set of user-defined models. Traditional DCM models the inputs of an experimental design; stimuli are treated as perturbations within a system, whereas sDCM (Daunizeau et al., 2009) relies on the modeling of hidden inputs. Given that PSSI is a task-agnostic measure of dynamic regulation of a control system, in which stochastic inputs are assumed, sDCM was better suited than standard DCM in identifying consistent models for PSSI results.

ROI definition. Our ROIs within the prefrontal-limbic threat circuit were defined from a synthesis of our present results, previous reports, and the relevant literature. We chose the IFG based upon group differences in PSSI $\beta$-signatures (see Results). We chose the ventromedial prefrontal cortex (vmPFC) based upon previous reports of fear generalization of which activation tracks safety signals in healthy individuals (Greenberg et al., 2013b), yet with a significantly lesser degree in individuals with GAD, therefore presenting evidence for inefficient safety-threat discrimination and overgeneralization of fear (Greenberg et al., 2013a). Finally, we chose the amygdala for its well-established role in threat processing (LeDoux, 2003) and for its relationship with the vmPFC inhibition (Motzkin et al., 2015).

The IFG was defined by the PSSI differences between patients and healthy controls reported here: a 6 -mm-radius sphere at [MNI: $-52,20$, 6]. The vmPFC was defined by the present PPI results, using a $6-\mathrm{mm}$ radius sphere at [MNI: $-10,40,-16$ ]. Given the strong habituation effect we previously reported (Greenberg et al., 2013b) within this context and the lack of PPI-specific correlations with IFG $\beta$, we used anatomical masks for the amygdala (left and right separately) based on the standard atlas (automated anatomical labeling toolbox) (TzourioMazoyer et al., 2002). Time-series for sDCM were obtained by using SPM8 to compute the first principal component from all voxels within each ROI, and were adjusted for the effects of interest contrast as speci-

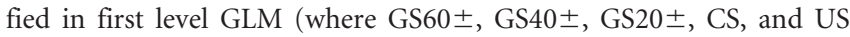
onsets were used to define conditions of interest).

Model specification, estimation, and comparison. We tested four models (see Fig. 5A) that differed in terms of their endogenous connections: (1) a fully connected model (bilateral connections between each pair of the three regions); (2) a unidirectional (top-down) serial model (the IFG modulates the vmPFC, which in turn modulates the amygdala); (3) a bidirectional serial model (adding bottom-up connections to Model 2); and (4) a unidirectional (top-down) parallel model (the IFG modulates the vmPFC and the amygdala in parallel). Based on the anatomy of this 
circuit, we hypothesized that all three ROIs would be fully connected (Model 1). As alternatives to the main model, we tested two models with serial connections from the IFG to the vmPFC to the amygdala (Models 2 and 3) based on the literature (Bishop, 2007, 2009; Johnstone et al., 2007; Hare et al., 2009). Each model consisted solely of intrinsic connections: no modulatory influences were tested (Almeida et al., 2009; Li et al., 2014). Models with the left or right amygdala along with the other ROIs were estimated separately because we had no a priori hypothesis. We did not consider models with bilateral amygdala to avoid complexity in model specification and interpretation. Therefore, separate models were estimated for each hemispheric amygdala.

BMS was used to determine the highest likelihood model from the given model set. We then extracted the individual connectivity strengths from the highest likelihood model and explored whether IFG PSSI was associated with this connectivity.

Probabilistic diffusion tractography

We hypothesized that structural connectivity within the prefrontal-limbic network would be associated with abnormal IFG $\beta$-signatures in participants with GAD. To this end, we investigated the relationship between IFG $\beta$-signatures and the integrity of the major prefrontal-limbic white matter pathway previously implicated in anxiety disorders (Kim and Whalen, 2009; Westlye et al., 2011; Hettema et al., 2012): the uncinate fasciculus (UF). We used standard preprocessing steps in FMRIB's Diffusion Toolbox (www.fmrib.ox.ac.uk/fsl): after skull stripping, we performed corrections for head motion and eddy currents via reference volume registration. Diffusion tensor parameters, such as fractional anisotropy (FA), were calculated by fitting a tensor model (DTIFIT) in FMRIB's Diffusion Toolbox. Finally, for probabilistic tractography, crossing fibers were modeled using Bayesian Estimation of Diffusion Parameters Obtained using Sampling Techniques in FMRIB Diffusion Toolbox (Behrens et al., 2007). As described previously (Cha et al., 2014a), we reconstructed the entirety of the UF using a global tractography approach, with tracts constrained by underlying anatomy or TRACULA (Yendiki et al., 2011). From the reconstructed UF (i.e., posterior distribution maps) in each individual, average FA values (per hemisphere) were derived. We then tested for correlations between IFG $\beta$-signatures and FA of the UF.

\section{Summary of statistics}

Group differences in $\beta$-signatures were examined using voxelwise two sample $t$-tests. Independent variables included group and subject, whereas individual $\beta$ images were used as dependent variables. To minimize bias, group differences in ACF b-values were examined using the same GLM approach and $t$-contrasts in SPM as in the PSSI group differences analysis. Pearson's correlation was used to test for an association between IFG $\beta$-signatures and MASQ-GDA anxiety subscales, connection strengths from sDCM, as well as FA of the UF. Partial correlations were used to control for confounding effects, as noted.

\section{Results}

IFG functional dynamics are more chaotic in GAD

Individuals with GAD showed PSSI significantly closer to white noise $(\beta=0)$, compared with healthy controls, in the left IFG
B

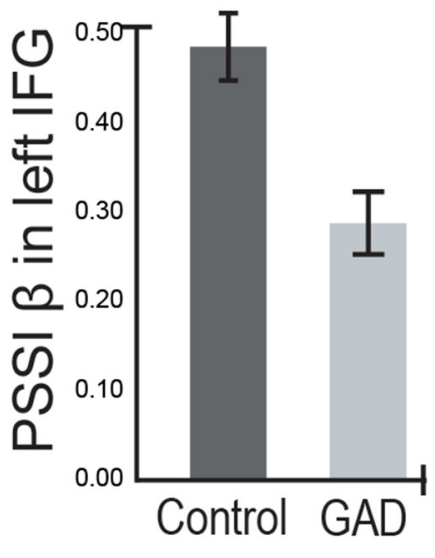

$\mathrm{D}$

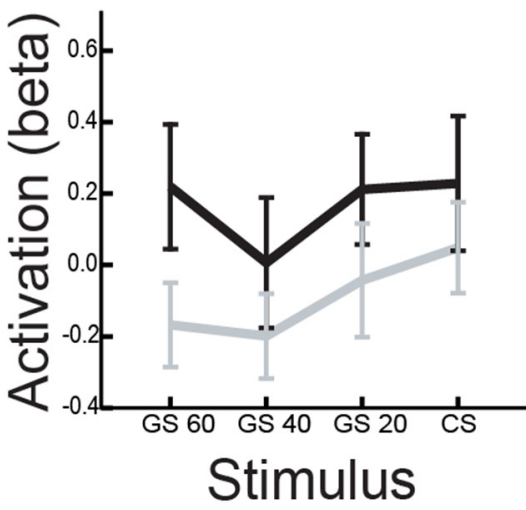

Figure 2. During fear generalization, individuals with GAD showed functional dynamics within the IFG that were significantly more chaotic (uncontrolled) than those of healthy controls. We quantified dynamics by power spectrum scale invariance $\beta$-signatures, which provide the slope of time-series fit to a power law, as well as autocorrelation lifetime $\tau$, which indicates

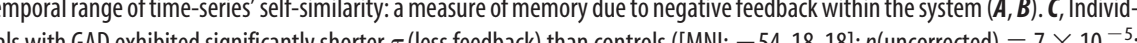
cluster extent $=57 ; p$ (corrected) $<0.001)$ for the same region. $D$, Activation of the same IFG (from a 6-mm-radius sphere centered controls. Further investigation revealed that the decreased activation in the IFG was independent from the decreased PSSI $\beta$-signatures or autocorrelation lifetime $\tau$.

ROI at corrected $p<0.02\left(\mathrm{MNI}:-52,20,6\right.$; peak $t_{(55)}=4.46$ cluster extent $=57$ voxels, at a voxelwise $p$ threshold of 0.005 ) $\left(\beta_{\text {controls }}=0.45 \pm 0.21 ; \beta_{\mathrm{GAD}}=0.28 \pm 0.18\right.$; Fig. $\left.2 A, B\right)$. No significant group differences were found in the right IFG $(p>$ $0.05)$. In an exploratory analysis, the vPFC and amygdala showed no significant group differences in PSSI $\beta$-signatures (ROIcorrected $p$ values $>0.05$ ).

Furthermore, the mean autocorrelation lifetime $\tau$ (s) of IFG activity was significantly reduced in GAD versus healthy controls at corrected $p<0.05\left(\tau_{\mathrm{HC}}=2.88 \mathrm{~s}, \tau_{\mathrm{GAD}}=2.50 \mathrm{~s}\right.$; peak $t_{(55)}=$ 4.12; peak $p=0.00007$; Fig. $2 C$ ). These results indicate that the IFG BOLD time-series are more chaotic in individuals with GAD compared with healthy controls, suggesting either stronger excitatory chaotic inputs or weaker constraints imposed by negative (inhibitory) feedback within the system (Rădulescu and MujicaParodi, 2014). We found no significant differences between individuals with GAD and those comorbid with major depressive disorder ( $p=0.43$, two-sample $t$ test). The group difference remained significant after controlling for two in-scanner head motion parameters (peak and mean framewise displacement); the effect of head motion on the PSSI $\beta$-signatures in the left IFG were also non-significant ( $p$ values $>0.59$ ). 
Table 3. Model selection results with PSSI $\beta$-signatures in the left IFG as the dependent variables and $\mathrm{MASQ}^{a}$

\begin{tabular}{|c|c|c|c|c|c|c|}
\hline \multirow[b]{2}{*}{ Model } & \multirow[b]{2}{*}{ Predictors } & \multicolumn{2}{|c|}{$\begin{array}{l}\text { Goodness of fit } \\
\text { (in small-is-better form) }\end{array}$} & \multicolumn{3}{|c|}{ Omnibus test } \\
\hline & & $\begin{array}{l}\text { Log } \\
\text { likelihood }\end{array}$ & $\begin{array}{l}\text { Akaike's } \\
\text { Information } \\
\text { criterion }\end{array}$ & $\begin{array}{l}\text { Likelihood } \\
\text { ratio } \chi^{2}\end{array}$ & df & $p$ \\
\hline \multicolumn{7}{|l|}{ Model 1: GLM } \\
\hline 1 & $\begin{array}{l}\text { GDA, GDD, AA, } \\
\quad A D\end{array}$ & 14.41 & -16.83 & 9.478 & 5 & 0.05 \\
\hline 2 & GDA, GDD, AD & 14.22 & -18.43 & 9.08 & 3 & 0.028 \\
\hline 3 & GDA, GDD & 13.42 & -18.84 & 7.496 & 2 & 0.024 \\
\hline Component & Variance & Error & $R^{2}$ & PRESS & \multicolumn{2}{|c|}{$\begin{array}{l}\text { Predicted } R^{2} \\
\text { by L00CV }\end{array}$} \\
\hline
\end{tabular}

Model 2: PLS

\begin{tabular}{llllll} 
regression & & & & & \\
1 & 0.354 & 0.693 & 0.251 & 0.910 & 0.016 \\
2 & 0.817 & 0.670 & 0.276 & 0.893 & 0.035 \\
\hline
\end{tabular}

${ }^{a} A A$, Anxious arousal; $A D$, anhedonic depression; PRESS, prediction residual error sum of squares; $L 00 C V$, leave-oneout cross validation.

We then asked whether the decreased PSSI $\beta$-signatures (i.e., more chaotic BOLD time-series) in the left IFG merely reflected reduced overall activation. To test this, we performed a GLM analysis on the IFG region defined by the PSSI analyses (the IFG ROI was defined as a 6-mm-radius sphere centered upon peak coordinates for PSSI group differences) (Fig. 2D). Repeatedmeasures ANOVA using stimulus as the within-subject variable, group as the between-subject variable, and age as the covariate, revealed a significant effect of group $(F=8.35, p=0.006)$ in this left IFG cluster, but a nonsignificant effect of stimulus $(F=0.61$, $p=0.61$ ). However, in our control analyses, we found no evidence for a significant association between the decreased IFG activation estimates and the IFG PSSI $\beta$-signatures (GLM using PSSI $\beta$-signatures as the dependent variable, group as the independent variable, mean IFG activation across stimuli as the covariate: $p=0.34$; repeated-measures ANOVA using IFG activation estimates as the dependent variable, group as the between-subject variable, IFG PSSI $\beta$ as the covariate: $p=0.37)$. Generalization effects were nonsignificant in either group ( $p$ values $>0.4$; linear trend analysis in repeated-measures of ANOVA). These results suggest that PSSI $\beta$-signatures for the IFG BOLD time-series in GAD do not result from simple differences in task-induced activation.

We then tested whether PSSI $\beta$-signatures in the left IFG correlate with anxiety (GDA) and depression (general distress depression [GDD]) symptom measures within the GAD group. In stepwise backward selection, the model with GDA and GDD showed the best goodness of fit (Table 3). Interestingly, whereas GDA showed a negative association with the PSSI $\beta$-signatures, GDD showed a positive association (GDA: coefficient = $-0.008 \pm 0.0037(\mathrm{SE}) ; p=0.022$; GDD: coefficient $=0.008 \pm$ $0.0026(\mathrm{SE}) ; p=0.003)$. No additional benefit was derived from entering the confounding variables (i.e., age, average IFG activation induced by stimuli, two in-scanner head motion parameters). No collinearity between GDA and GDD was detected (variance inflation factor $=1.24$ ). Interaction between the two was nonsignificant $(p>0.92)$.

Although the negative association of anxiety symptoms with PSSI IFG was anticipated, the positive association of a depression symptom was not. We further tested whether or not these findings were merely driven by spurious parametric effects between the two particular symptom measures. To this end, we performed partial least square regression that allowed us to extract orthogonal components from multiple dependent variables, that is, all four subscales of MASQ. Two components were selected as significant predictors of the PSSI $\beta$ values (Table 3 ): these two components account for $28 \%$ of the variance of the PSSI $\beta$ values (Fig. $3 B$ ). In this model, two anxiety symptoms (GDA and anxious arousal) showed negative coefficients, whereas the other two depression symptoms (GDD and anhedonic depression) showed positive coefficients. Together, these results suggest that decreased IFG PSSI values are associated with increased anxiety symptoms and decreased depression symptoms.

\section{IFG $\boldsymbol{\beta}$-signatures correlate with effective connectivity between prefrontal and limbic regions}

We investigated whether prefrontal-limbic connectivity correlated with the observed PSSI $\beta$-signatures in the left IFG. As motivated by previous work (Greenberg et al., 2013a; Cha et al., 2014a), our ROIs included the vmPFC (including medial and lateral aspects) and amygdala.

We first used PPI analysis to test whether task-induced effective connectivity with the IFG, modulated by generalization stimuli, correlates with IFG PSSI $\beta$-signatures. In the ROI analysis, we found that more chaotic IFG $\beta$-signatures were associated with weaker left IFG connectivity to the left vmPFC and right ventrolateral prefrontal cortex, but not to the amygdala (Fig. 4; Table 2). In the whole-brain analysis, we found positive correlations between IFG $\beta$-signatures and IFG connectivity with caudate, and the mid-temporal lobes at corrected $p$ values $<0.05$. No group differences in IFG-seeded functional connectivity were observed ( $p$ values $>0.1$ ). These findings suggest that IFG $\beta$-signatures result from the IFG's interactions within the prefrontal-limbic threat circuit.

PSSI $\beta$-signatures represent feedback dynamics of task-free data. Therefore, we further examined task-free functional (effective) connectivity using sDCM. We examined the causal model explaining the IFG's interactions with the vmPFC and amygdala (for ROI definition, see Materials and Methods). We tested four models representing a synthesis of the present results and our previous work highlighting the roles of the IFG (Tolkunov et al., 2010; Mujica-Parodi et al., 2014), vmPFC (Cha et al., 2014a), and amygdala (Tolkunov et al., 2010; Rădulescu and Mujica-Parodi, 2014).

Because we had no a priori hypothesis on the laterality of the amygdala, whereas the IFG and the vmPFC ROIs were in the left hemisphere, we tested four tripartite models comprising the left IFG, left vmPFC, and either the left or the right amygdala separately. Notably, only the models with the right amygdala successfully converged in our model estimation procedure. We therefore proceeded to Bayesian model comparison only using the four sDCMs with the right amygdala and the other ROIs.

BMS indicated that the fully connected model best supported BOLD time-series of the IFG-vmPFC-amygdala network (Fig. 5; Table 4). We found significant correlations between IFG $\beta$-signatures and vmPFC $\rightarrow$ IFG connectivity $(r=0.37, p=$ $0.007)$ and IFG $\rightarrow$ vmPFC connectivity $(r=0.43, p=0.001)$. Here, the greater the positive bidirectional connectivity between the two regions, the closer the left IFG $\beta$-signatures were to pink noise. The correlation between IFG $\rightarrow$ vmPFC and vmPFC $\rightarrow$ IFG connectivity was significant $(r=0.95, p<0.001)$, indicating a highly reciprocal relationship. BMS selected the fully connected model for both GAD and healthy controls, and there were no group differences in connection strength for any of the six connections (two-sample $t$ test, $p$ values $\geq 0.1$ ). Internode connec- 
A

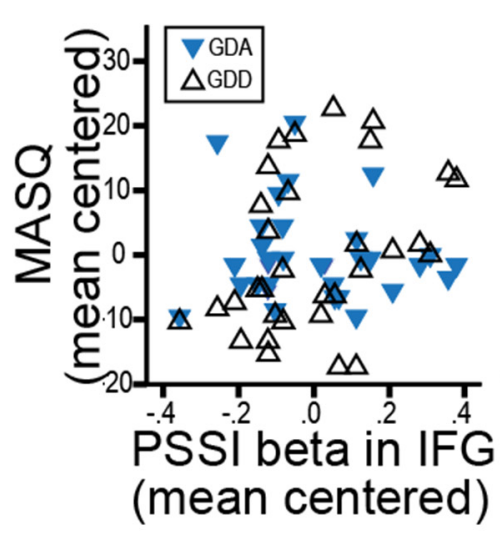

B

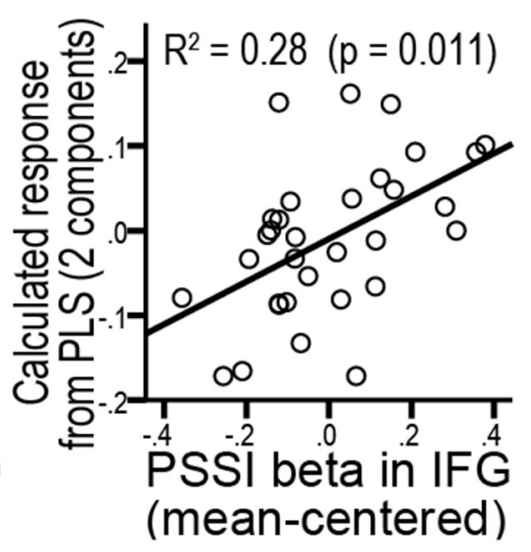

C

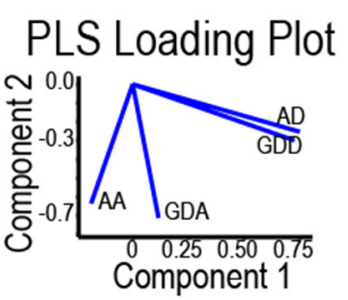

PLS Coefficient Plot

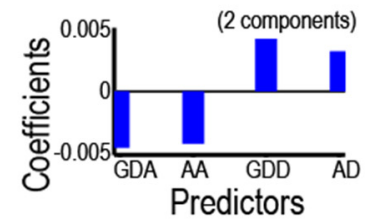

Figure 3. Power law $\beta$-signatures of the IFG are associated with symptoms of anxiety and depression. $\boldsymbol{A}$, Scatter plot between PSSI $\beta$ values versus GDA and GDD. GDA and GDD were selected as significant predictors of PSSI $\beta$ (among four subscales of MASQ; see Results) (Watson et al., 1995). B, C, Partial Least Square (PLS) regression showing that two orthogonal factors (components) extracted from MASQ subscales predict PSSI $\beta$ values. B, Scatter plot between calculated response from PLS model and PSSI $\beta$ values. C, PLS loading plot and coefficient plot. In the loading plot, two depression symptoms (anhedonic depression, GDD) were significantly separated from two anxiety symptoms (anxious arousal, GDA). In the coefficient plot, anxiety and depression symptoms showed negative and positive coefficients, respectively.

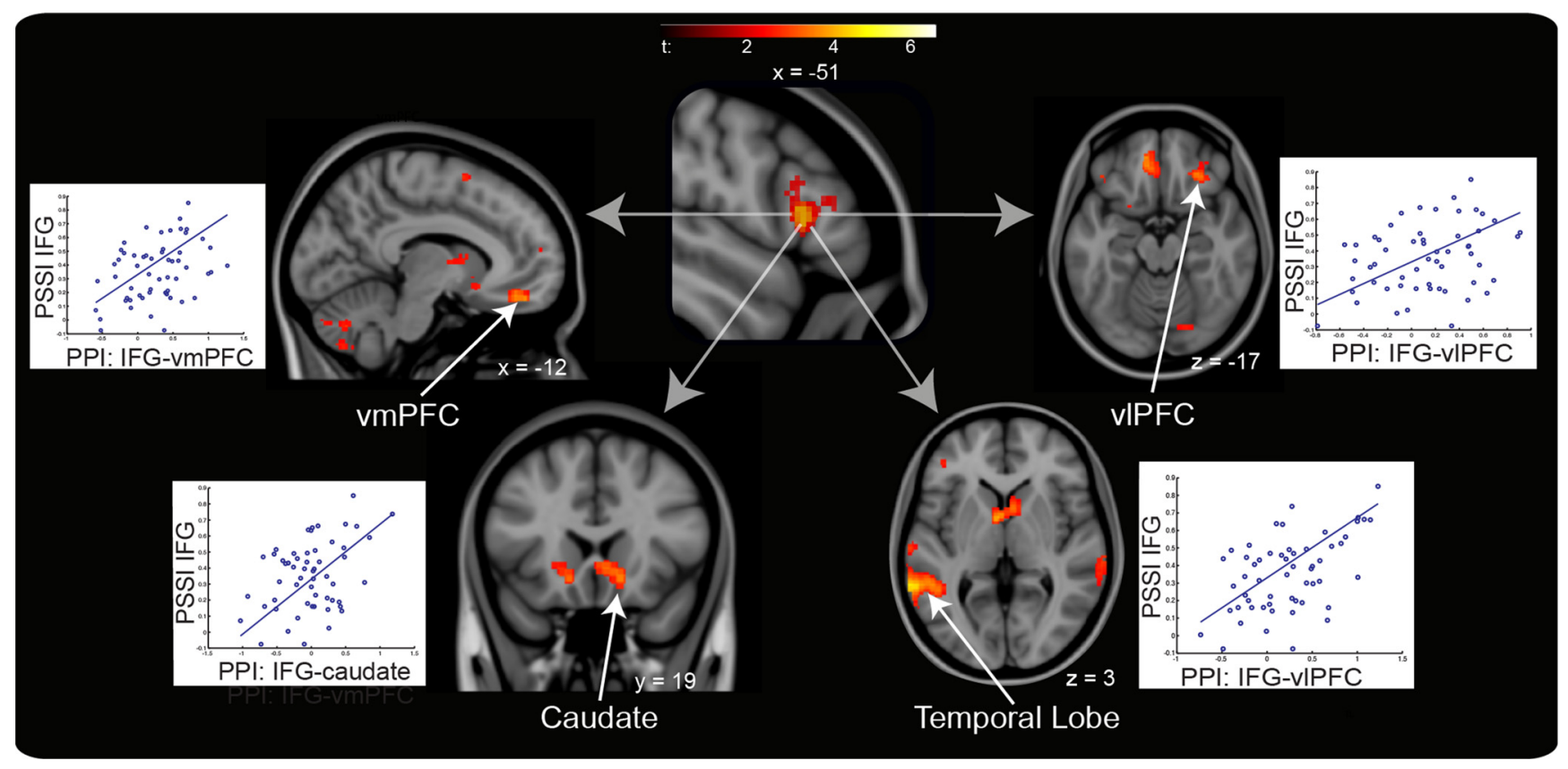

Figure 4. IFG PSSI $\beta$-signatures correlate with effective connectivity modulated by GS. IFG PSSI $\beta$-signatures significantly correlated with the GS-modulated IFG connectivity (differences compared with the baseline) between the IFG-seed region and the vmPFC, ventrolateral prefrontal cortex (vIPFC), caudate, and temporal lobe. Results were corrected for multiple comparison by estimating cluster extents under a voxelwise $p$ threshold of 0.005 that correspond to $\alpha$ of 0.05 (see Materials and Methods); for presentational purposes only, a $p$ threshold of 0.005 with cluster extents of 10 voxels were used here.

tion strengths ranged from $-0.005 \mathrm{~Hz}(\mathrm{vmPFC} \rightarrow$ amygdala) to $0.06 \mathrm{~Hz}$ (IFG $\rightarrow$ amygdala), and all connection probabilities reached significance within the sDCM construct (connection probability $\simeq 1$ ), consistent with values cited in the literature (Ma et al., 2015).

IFG regulation (PSSI) correlates with fiber integrity of the prefrontal-limbic white matter pathway

Finally, we tested whether the observed $\beta$-signatures were associated with integrity of the major white matter pathway connecting the IFG with the prefrontal-limbic threat circuit (e.g., the vmPFC and amygdala). As shown in Figure $6 A$, a representative reconstructed UF appeared to connect the IFG with the vmPFC and amygdala in the left hemisphere. We found a significant positive correlation between FA of the UF and IFG $\beta$-signatures $(r=0.30 ; p=0.03$; Fig. 6). White matter integrity showed nonsignificant correlation with anxiety or depression subscales ( $p$ values $>0.7$ ), although we previously reported a trend toward lower FA in participants with GAD compared with controls within this dataset (Cha et al., 2014a). Nonetheless, this effect was significant after controlling for the effects of group, anxiety (MASQ-GDA), and depression (MASQGDD; $r=0.28, p=0.05)$. These results suggest that the dynamics within the prefrontal limbic circuit reflect not only functional, but also structural, connectivity.

Since we found that $\beta$-signatures in the IFG are positively correlated with IFG functional connectivity within the 
A

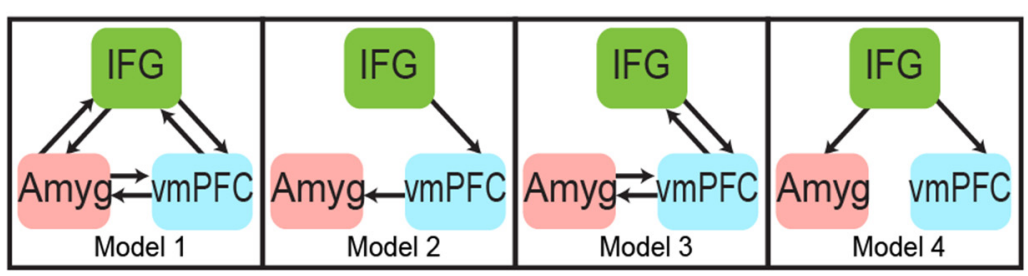

B

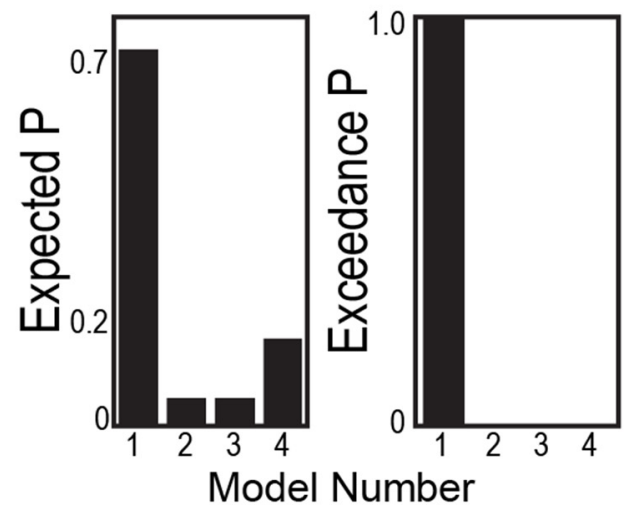

C

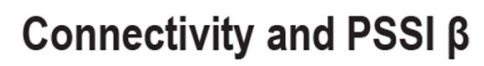

Figure 5. sDCM suggests the prefrontal-limbic threat circuit consisting of the IFG, vmPFC, and amygdala during fear generalization. $\boldsymbol{A}$, Model space. $\boldsymbol{B}$, In Bayesian model selection, Model 1 (a fully connected model) showed the greatest expected probability and exceedance probability. C, Individual variability in bidirectional connectivity between the IFG and vmPFC significantly correlates with IFG PSSI $\beta$-signatures, showing stronger connectivity as $\beta$-signatures shift to pink noise, and weaker connectivity as $\beta$-signatures shift to white noise. ${ }^{* *} p<0.01$. ${ }^{* *} p<0.001$.

Table 4. SDCM connection strengths for a fully connected tripartite model, consisting of the IFG, vmPFC, and amygdala ${ }^{a}$

\begin{tabular}{lc}
\hline Connection & Strength $(\mathrm{Hz})$ \\
\hline Left IFG $\rightarrow$ Left vmPFC & 0.02 \\
Left IFG $\rightarrow$ Right amygdala & 0.06 \\
Left vmPFC $\rightarrow$ Left IFG & 0.02 \\
Left vmPFC $\rightarrow$ Right amygdala & -0.005 \\
Right amygdala $\rightarrow$ Left IFG & 0.03 \\
Right amygdala $\rightarrow$ Left vmPFC & 0.01 \\
\hline
\end{tabular}

${ }^{a}$ We obtained the connection parameters from Bayesian fixed-effect analysis average, based on our assumption that optimal model structure is identical across subjects and groups (Stephan et al., 2010). All connections were significantly greater than 0 (posterior $p$ values $=1$ ). No group differences were found ( $p$ values $>0.1$ ).

prefrontal-limbic circuit, we tested whether greater fiber integrity of the UF is correlated with greater functional connectivity within this circuit. We found a significant correlation between the IFGvmPFC PPI connectivity modulated by GS and fiber integrity (Pearson's $r=0.22, p=0.05$, one-sided).

\section{Discussion}

We found that aberrant IFG dynamics replicated results that we first identified for trait anxiety (Tolkunov et al., 2010) in both clinical anxiety and with anxious symptoms identified by the MASQ (suggesting an anxiety spectrum approach to be appropriate) and that this shift in functional dynamics was associated with altered functional and structural prefrontal-limbic connectivity within the circuit comprising the IFG, vmPFC, and amygdala. Specifically, IFG functional dynamics were strongly associated with bidirectional interactions with the vmPFC, whereas the vmPFC exhibited inhibitory causal influence upon the amygdala, a well-established excitatory node within the prefrontal-limbic network (LeDoux, 2003).

Using modeling and simulations, we previously showed that dynamics for a node within a negative feedback loop become more chaotic, or "white," under two conditions: when excitatory inputs are strengthened or when negative feedback is weakened (Rădulescu and Mujica-Parodi, 2014). This study provides converging evidence for the latter and supports a role for the IFG that is indirectly inhibitory (via interactions with the vmPFC, which inhibits the amygdala) rather than directly inhibiting the amygdala. This prefrontallimbic pathway is consistent with nonhuman primate tracing, which shows extensive anatomical connections from the IFG to the vmPFC, which in turn innervates to and from subcortical limbic areas (Barbas and Pandya, 1989). In our task, we used Pavlovian fear conditioning to induce fear to a particular stimulus (CS), and then presented stimuli across a gradient of perceptual similarity to that stimulus; these ranged from identical (CS) to clearly distinct $( \pm 60 \%)$, with more ambiguous stimuli in between. We previously showed that, for healthy individuals, both the vmPFC and ventral tegmental area closely track the gradient of perceptual similarity to the CS. The ventral tegmental area is strongly activated to the CS, and becomes gradually less activated as cues show greater dissimilarity to the CS (Cha et al., 2014b). In contrast, the vmPFC follows the opposite pattern, showing strongest activation to the cue most dissimilar to the CS $( \pm 60 \%)$, and becomes gradually less activated as cues show greater similarity to the CS (Greenberg et al., 2013a). These patterns are consistent with the ventral tegmental area's role reinforcement learning in response to positive or, in the present case, negative reward (Cha et al., 2014b; Hennigan et al., 2015), and also suggests that the vmPFC's role may be primarily inhibitory (Motzkin et al., 2015) (i.e., the "brakes" are off when the cue indicates alarm, but are activated in direct proportion to the likelihood that the cue is deemed "safe"). This interpretation of the GLM analyses is supported by our current sDCM results (Table 3 ), which indicated that the only inhibitory connectivity observed was from the vmPFC to the amygdala.

Of note, only sDCMs containing the right amygdala converged successfully, but not the ones with the left amygdala. This suggests that we have no basis to infer significant influence by the left amygdala in this context. The involvement of the right amygdala, but not left, in the inhibitory vmPFC during threat processing seems consistent with previous literature demonstrating the role of the vmPFC inhibition on the right amygdala. A human fear extinction study with functional neuroimaging suggests the inhibitory role of the left vmPFC (or subgenual anterior cingulate cortex; MNI: $-4,31,-6$ ) onto activation of the right amygdala (MNI: 20, $-6,-15)$, but not the left (Phelps et al., 2004). A recent study of individuals with bilateral vmPFC lesions showed the hyperactive right amygdala, not left, to aversive stimuli, suggesting that the vmPFC inhibition may exert preferentially, if not exclusively, on the right amygdala (Motzkin et al., 2015). Together, our results suggest that prefrontal threat processing, including threat-safety assessment, decision making, and regulation, may occur in the left hemisphere preferentially, if not exclusively, and that inhibition of the amygdala may be preferen- 
A

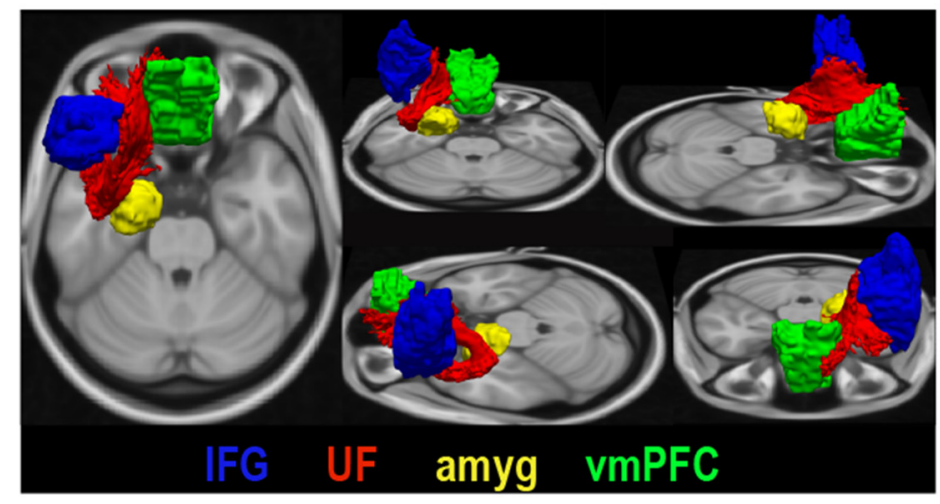

B

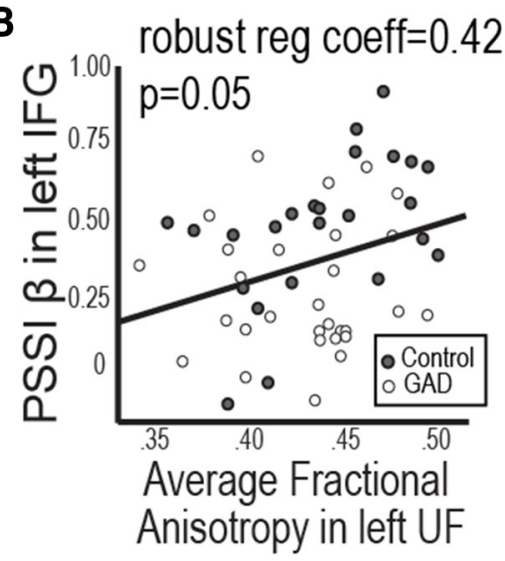

Figure 6. PSSI $\beta$-signatures of the IFG correlate with fiber integrity of the uncinate fasciculus. $\boldsymbol{A}$, The uncinate fasciculus as a potential anatomical substrate of IFG's interaction with the prefrontal-limbic threat circuit. A representative probabilistic tractography result (posterior distribution) shows the uncinate fasciculus (red) connecting the IFG (blue) with the vmPFC/orbitofrontal cortex (green) and the amygdala (yellow). The subcortical/cortical masks were derived from segmentation and parcellation analyses in Freesurfer (http://freesurfer.net/). $\boldsymbol{B}, \beta$-Signatures of the IFG significantly correlate with fiber integrity, indexed by FA, of the uncinate fasiculus. robust reg coeff, Robust regression coefficient.

tially on the right hemisphere. Nevertheless, the laterality of such effects or interhemispheric prefrontal-amygdala connections requires further investigation.

But how does the brain decide what is "safe" with respect to perceptually ambiguous threat? Here, the IFG seems to be playing a key role. Looking at a spectrum of threat assessment that included 137 individuals, across three independent studies $(N=57$ for our current study of clinical anxiety; $N=50$ for our study of trait anxiety, Tolkunov et al., 2010; and $N=30$ for our study of "reckless" risk-takers, Mujica-Parodi et al., 2014), we observe a clear inverted-U pattern for Brodmann's area 45, a subset of the IFG. Individuals at the center of the threat assessment spectrum (showing accurate perception of threat: physiologically in anticipation of jumping out of a plane, behaviorally in classifying faces with ambiguous affect, as well as emotionally with self-reported low trait anxiety in nondangerous contexts) showed IFG regulation in the "pink-noise" range, which our simulations show to occur when a control system includes optimal feedback (Rădulescu and Mujica-Parodi, 2014), and which our sDCM results in the current study linked with strong bidirectional connectivity to the vmPFC. However, individuals at both ends of the spectrum (exceptionally anxious and exceptionally reckless) showed circuit-wide dysregulation localized most strongly to the IFG, with chaotic "white-noise" dynamics. Individuals at each end of the spectrum would appear to be opposites of one another (our clinically anxious sample identified threat where it did not exist, whereas our reckless sample failed to identify threat where it did exist), yet the most prominent feature that they both had in common was a failure to accurately assess ambiguous threat. The fact that both ends of the spectrum exhibit a disconnect between the IFG and the rest of the prefrontal-limbic circuit suggests that the IFG's role in fear regulation is indirect, potentially by providing disambiguation of ill-defined stimuli, which informs the (inhibitory) vmPFC in discriminating threat versus safety cues. Our interpretation is consistent with our sDCM results, which provide evidence for a fully connected (closed-circuit) model, with the altered IFG dynamics found in anxious individuals most strongly reflecting interactions between the IFG and vmPFC, but not between the IFG and amygdala, or between the vmPFC and amygdala.

The dissociation of roles within the circuit is also supported by the wider human neuroimaging literature. A Neurosynth (www.neurosynth.org) meta-analysis of $272 \mathrm{fMRI}$ studies of "fear" alone implicates the vmPFC $(Z=4.67 ; x=0, y=44$, $z=2$ ) but not the IFG, whereas $94 \mathrm{fMRI}$ studies of "ambiguous" cues alone implicate the left IFG $(Z=4.68 ; x=46, y=32, z=10)$ but not the vmPFC. Likewise, the IFG is most commonly implicated not only in the ambiguity of fear (Bach et al., 2009), but also in ambiguity of semantics (Bozic et al., 2010; Rodd et al., 2012). One theory that has been proposed (Roy et al., 2012) is that the vmPFC acts as a hub that itself establishes "meaning" by processing inputs from various accessory regions that provide cognitive contexts (such as disambiguation of stimulus percepts), and thereby elicits appropriate inhibitory control. The classical view of the prefrontal-limbic system is that the amygdala provides a "coarse-grained" threat assessment, with further "fine-grained" information, processed cortically, either amplifying or ameliorating the initial assessment (LeDoux, 2003). The fact that we see IFG dysregulation at both ends of the threat-assessment spectrum suggests that the IFG may process ambiguous stimuli by providing a set-point or filter in terms of informational signalto-noise ratios: assessing whether sufficient data have been acquired to trigger a decision to the vmPFC that a given stimulus is of one type or another; in this case, either threatening or benign. This hypothesis, if correct, would explain how both ends of the threat-assessment spectrum might share dysregulation of the IFG and its interactions with the vmPFC, amygdala, and associated feedback. If more anxious individuals' IFG thresholds for informational signal-to-noise ratios are set too high, threat assessment remains at the level of the initial coarse-grained excitatory amygdala response, without the full benefit of being informed by the fine-grained cortical information required for inhibition. On the other hand, if reckless individuals' IFG thresholds are set too low, subsequent inhibition of the initial coarse-grained excitatory response will be triggered, even in the absence of much-needed fine-grained cortical information. Future work will be necessary to test this hypothesis.

Our multimodal MRI approach identified a potential anatomical substrate of the IFG's interaction with the vmPFC, amygdala, as well as other regions of the prefrontal-limbic network. The estimated UF in this study is poised at the mid-point between the vmPFC and the amygdala, suggesting that the UF may underlie direct synaptic contact among these regions. The significant correlation between the fiber integrity of the UF and 
the PSSI of the IFG further suggests IFG's regulatory role within the prefrontal-limbic threat circuit is closely related to the integrity of its anatomical substrate. This discovery presents novel anatomical validation of the IFG PSSI measures in relation with the prefrontal-limbic connectivity.

We previously reported a marginally significant decrease in mean FA of the UF in participants with GAD compared with healthy controls $(p=0.073$ ) (Cha et al., 2014b). Given that our participants with GAD were never medicated and were relatively young (age $22 \pm 5.2$ years), it is possible that integrity of the UF may decrease further over time, bringing our findings in line with previous studies of GAD (Tromp et al., 2012). On the other hand, our IFG PSSI $\beta$-signatures showed a significant decrease in GAD. One possible interpretation is that PSSI $\beta$-signatures representing prefrontal-limbic dynamics or feedback mechanisms is more sensitive than structural connectivity of the prefrontal-limbic pathway. Alternatively, it is possible that our measure of structural connectivity in this study (mean FA of the entire UF) is not sufficiently sensitive to identify these specific effects.

The current study has several important directions for future work. First, this study was performed only on females; therefore, it will be important to replicate results in males. However, given that our previous findings of personality differences in trait anxiety within a healthy adult population of both males and females $(N=50)$ also found that anxiety correlated with IFG PSSI $\beta$-signatures (Tolkunov et al., 2010), we expect the similar effect of abnormally decreased IFG PSSI $\beta$-signatures in males with GAD. Second, although we provide evidence that the IFG appears to play a key role in the evaluation of ambiguous threat, we remain unclear as to the precise mechanism by which this evaluation occurs differentially at both ends of the hyperarousal and hypoarousal spectrum with respect to its interactions within the full control circuit. Finally, this type of systems-based approach may not only provide a valuable role in identifying general dimensional measures, as per current Research Domain Criteria, but also in making possible neuroimaging-driven computational modeling of functional circuits that explicitly include nonlinear feedback. In particular, the latter may provide neurodevelopmental insight into the mechanisms by which different types of dysregulation in brain control systems may predict clinical trajectories for mental disorders.

\section{References}

Almeida JR, Versace A, Mechelli A, Hassel S, Quevedo K, Kupfer DJ, Phillips ML (2009) Abnormal amygdala-prefrontal effective connectivity to happy faces differentiates bipolar from major depression. Biol Psychiatry 66:451-459. CrossRef Medline

American Psychiatric Association (2000) Diagnostic and statistical manual of mental disorder (Ed 4). Washington, DC: American Psychiatric Association Task Force on DSM-IV.

Aron AR, Fletcher PC, Bullmore ET, Sahakian BJ, Robbins TW (2003) Stopsignal inhibition disrupted by damage to right inferior frontal gyrus in humans. Nat Neurosci 6:115-116. CrossRef Medline

Bach DR, Seymour B, Dolan RJ (2009) Neural activity associated with the passive prediction of ambiguity and risk for aversive events. J Neurosci 29:1648-1656. CrossRef Medline

Barbas H, Pandya DN (1989) Architecture and intrinsic connections of the prefrontal cortex in the rhesus monkey. J Comp Neurol 286:353-375. CrossRef Medline

Behrens TE, Berg HJ, Jbabdi S, Rushworth MF, Woolrich MW (2007) Probabilistic diffusion tractography with multiple fibre orientations: what can we gain? Neuroimage 34:144-155. CrossRef Medline

Bishop SJ (2007) Neurocognitive mechanisms of anxiety: an integrative account. Trends Cogn Sci 11:307-316. CrossRef Medline

Bishop SJ (2009) Trait anxiety and impoverished prefrontal control of attention. Nat Neurosci 12:92-98. CrossRef Medline
Bishop S, Duncan J, Brett M, Lawrence AD (2004) Prefrontal cortical function and anxiety: controlling attention to threat-related stimuli. Nat Neurosci 7:184-188. CrossRef Medline

Bozic M, Tyler LK, Ives DT, Randall B, Marslen-Wilson WD (2010) Bihemispheric foundations for human speech comprehension. Proc Natl Acad Sci U S A 107:17439-17444. CrossRef Medline

Cha J, Greenberg T, Carlson JM, Dedora DJ, Hajcak G, Mujica-Parodi LR (2014a) Circuit-wide structural and functional measures predict ventromedial prefrontal cortex fear generalization: implications for generalized anxiety disorder. J Neurosci 34:4043-4053. CrossRef Medline

Cha J, Carlson JM, Dedora DJ, Greenberg T, Proudfit GH, Mujica-Parodi LR (2014b) Hyper-reactive human ventral tegmental area and aberrant mesocorticolimbic connectivity in overgeneralization of fear in generalized anxiety disorder. J Neurosci 34:5855-5860. CrossRef Medline

Clauset A, Shalizi CR, Newman MEJ (2009) Power law distributions in empirical data. Siam Rev 51:661-703. CrossRef

Daunizeau J, Friston KJ, Kiebel SJ (2009) Variational Bayesian identification and prediction of stochastic nonlinear dynamic causal models. Physica D 238:2089-2118. CrossRef Medline

Depue BE, Curran T, Banich MT (2007) Prefrontal regions orchestrate suppression of emotional memories via a two-phase process. Science 317: 215-219. CrossRef Medline

Eippert F, Veit R, Weiskopf N, Erb M, Birbaumer N, Anders S (2007) Regulation of emotional responses elicited by threat-related stimuli. Hum Brain Mapp 28:409-423. CrossRef Medline

First MB, Spitzer RL, Gibbon M, Williams JBW (2002) Structured clinical interview for DSM-IV-TR axis I disorders, research version, patient edition. New York: Biometrics Research, New York State Psychiatric Institute.

Fitzgerald DA, Angstadt M, Jelsone LM, Nathan PJ, Phan KL (2006) Beyond threat: amygdala reactivity across multiple expressions of facial affect. Neuroimage 30:1441-1448. CrossRef Medline

Friston KJ, Buechel C, Fink GR, Morris J, Rolls E, Dolan RJ (1997) Psychophysiological and modulatory interactions in neuroimaging. Neuroimage 6:218-229. CrossRef Medline

Friston KJ, Harrison L, Penny W (2003) Dynamic causal modelling. Neuroimage 19:1273-1302. CrossRef Medline

Greenberg T, Carlson JM, Cha J, Hajcak G, Mujica-Parodi LR (2013a) Ventromedial prefrontal cortex reactivity is altered in generalized anxiety disorder during fear generalization. Depress Anxiety 30:242-250. CrossRef Medline

Greenberg T, Carlson JM, Cha J, Hajcak G, Mujica-Parodi LR (2013b) Neural reactivity tracks fear generalization gradients. Biol Psychol 92:2-8. CrossRef Medline

Hare TA, Camerer CF, Rangel A (2009) Self-control in decision-making involves modulation of the vmPFC valuation system. Science 324:646648. CrossRef Medline

He BJ (2011) Scale-free properties of the functional magnetic resonance imaging signal during rest and task. J Neurosci 31:13786-13795. CrossRef Medline

He BJ, Zempel JM, Snyder AZ, Raichle ME (2010) The temporal structures and functional significance of scale-free brain activity. Neuron 66:353369. CrossRef Medline

Hennigan K, D’Ardenne K, McClure SM (2015) Distinct midbrain and habenula pathways are involved in processing aversive events in humans. J Neurosci 35:198-208. CrossRef Medline

Hettema JM, Kettenmann B, Ahluwalia V, McCarthy C, Kates WR, Schmitt JE, Silberg JL, Neale MC, Kendler KS, Fatouros P (2012) Pilot multimodal twin imaging study of generalized anxiety disorder. Depress Anxiety 29:202-209. CrossRef Medline

Johnstone T, van Reekum CM, Urry HL, Kalin NH, Davidson RJ (2007) Failure to regulate: counterproductive recruitment of top-down prefrontal-subcortical circuitry in major depression. J Neurosci 27:88778884. CrossRef Medline

Kim MJ, Whalen PJ (2009) The structural integrity of an amygdalaprefrontal pathway predicts trait anxiety. J Neurosci 29:11614-11618. CrossRef Medline

LeDoux J (2003) The emotional brain, fear, and the amygdala. Cell Mol Neurobiol 23:727-738. CrossRef Medline

Li B, Friston KJ, Liu J, Liu Y, Zhang G, Cao F, Su L, Yao S, Lu H, Hu D (2014) Impaired frontal-basal ganglia connectivity in adolescents with internet addiction. Sci Rep 4:5027. CrossRef Medline 
Ma L, Steinberg JL, Cunningham KA, Lane SD, Kramer LA, Narayana PA, Kosten TR, Bechara A, Moeller FG (2015) Inhibitory behavioral control: a stochastic dynamic causal modeling study using network discovery analysis. Brain Connect 5:177-186. CrossRef Medline

McLean CP, Asnaani A, Litz BT, Hofmann SG (2011) Gender differences in anxiety disorders: prevalence, course of illness, comorbidity and burden of illness. J Psychiatr Res 45:1027-1035. CrossRef Medline

Miezin FM, Maccotta L, Ollinger JM, Petersen SE, Buckner RL (2000) Characterizing the hemodynamic response: effects of presentation rate, sampling procedure, and the possibility of ordering brain activity based on relative timing. Neuroimage 11:735-759. CrossRef Medline

Motzkin JC, Philippi CL, Wolf RC, Baskaya MK, Koenigs M (2015) Ventromedial prefrontal cortex is critical for the regulation of amygdala activity in humans. Biol Psychiatry 77:276-284. CrossRef Medline

Mujica-Parodi LR, Korgaonkar M, Ravindranath B, Greenberg T, Tomasi D, Wagshul M, Ardekani B, Guilfoyle D, Khan S, Zhong Y, Chon K, Malaspina D (2009) Limbic dysregulation is associated with lowered heart rate variability and increased trait anxiety in healthy adults. Hum Brain Mapp 30:47-58. CrossRef Medline

Mujica-Parodi LR, Carlson JM, Cha J, Rubin D (2014) The fine line between 'brave' and 'reckless': amygdala reactivity and regulation predict recognition of risk. Neuroimage 103:1-9. CrossRef Medline

Ochsner KN, Gross JJ (2005) The cognitive control of emotion. Trends Cogn Sci 9:242-249. CrossRef Medline

Ochsner KN, Silvers JA, Buhle JT (2012) Functional imaging studies of emotion regulation: a synthetic review and evolving model of the cognitive control of emotion. Ann N Y Acad Sci 1251:E1-E24. CrossRef Medline

Phelps EA, Delgado MR, Nearing KI, LeDoux JE (2004) Extinction learning in humans: role of the amygdala and vmPFC. Neuron 43:897-905. CrossRef Medline

Rădulescu A, Mujica-Parodi LR (2014) Network connectivity modulates power spectrum scale invariance. Neuroimage 90:436-448. CrossRef Medline

Rodd JM, Johnsrude IS, Davis MH (2012) Dissociating frontotemporal contributions to semantic ambiguity resolution in spoken sentences. Cereb Cortex 22:1761-1773. CrossRef Medline

Roy M, Shohamy D, Wager TD (2012) Ventromedial prefrontal-subcortical systems and the generation of affective meaning. Trends Cogn Sci 16:147156. CrossRef Medline

Rubin D, Fekete T, Mujica-Parodi LR (2013) Optimizing complexity measures for FMRI data: algorithm, artifact, and sensitivity. PLoS One 8:e63448. CrossRef Medline

Sagaspe P, Schwartz S, Vuilleumier P (2011) Fear and stop: a role for the amygdala in motor inhibition by emotional signals. Neuroimage 55: 1825-1835. CrossRef Medline

Stephan KE, Penny WD, Moran RJ, den Ouden HE, Daunizeau J, Friston KJ (2010) Ten simple rules for dynamic causal modeling. Neuroimage 49: 3099-3109. CrossRef Medline

Strawn JR, Bitter SM, Weber WA, Chu WJ, Whitsel RM, Adler C, Cerullo MA, Eliassen J, Strakowski SM, DelBello MP (2012) Neurocircuitry of generalized anxiety disorder in adolescents: a pilot functional neuroimaging and functional connectivity study. Depress Anxiety 29:939-947. CrossRef Medline

Tolkunov D, Rubin D, Mujica-Parodi L (2010) Power spectrum scale invariance quantifies limbic dysregulation in trait anxious adults using fMRI: adapting methods optimized for characterizing autonomic dysregulation to neural dynamic time-series. Neuroimage 50:72-80. CrossRef Medline

Tromp DP, Grupe DW, Oathes DJ, McFarlin DR, Hernandez PJ, Kral TR, Lee JE, Adams M, Alexander AL, Nitschke JB (2012) Reduced structural connectivity of a major frontolimbic pathway in generalized anxiety disorder. Arch Gen Psychiatry 69:925-934. CrossRef Medline

Tzourio-Mazoyer N, Landeau B, Papathanassiou D, Crivello F, Etard O, Delcroix N, Mazoyer B, Joliot M (2002) Automated anatomical labeling of activations in SPM using a macroscopic anatomical parcellation of the MNI MRI single-subject brain. Neuroimage 15:273-289. CrossRef Medline

Vanderhasselt MA, Kühn S, De Raedt R (2013) 'Put on your poker face': neural systems supporting the anticipation for expressive suppression and cognitive reappraisal. Soc Cogn Affect Neurosci 8:903-910. CrossRef Medline

Watson D, Weber K, Assenheimer JS, Clark LA, Strauss ME, McCormick RA (1995) Testing a tripartite model: I. Evaluating the convergent and discriminant validity of anxiety and depression symptom scales. J Abnorm Psychol 104:3-14. CrossRef Medline

Westlye LT, Bjørnebekk A, Grydeland H, Fjell AM, Walhovd KB (2011) Linking an anxiety-related personality trait to brain white matter microstructure: diffusion tensor imaging and harm avoidance. Arch Gen Psychiatry 68:369-377. CrossRef Medline

Woolrich MW, Ripley BD, Brady M, Smith SM (2001) Temporal autocorrelation in univariate linear modeling of FMRI data. Neuroimage 14: 1370-1386. CrossRef Medline

Yendiki A, Panneck P, Srinivasan P, Stevens A, Zöllei L, Augustinack J, Wang R, Salat D, Ehrlich S, Behrens T, Jbabdi S, Gollub R, Fischl B (2011) Automated probabilistic reconstruction of white-matter pathways in health and disease using an atlas of the underlying anatomy. Front Neuroinform 5:23. CrossRef Medline 